\title{
Study of exotic mesons in pp and AA collisions
}

\author{
Mikhail Barabanov, Alexander Vodopyanov \\ Joint Institute for Nuclear Research, 141980 Joliot-Curie 6, Dubna, Russia 141980
}

\begin{abstract}
The possibility of study of exotic mesons is discussed. The paper includes detailed analysis of strong, weak and electromagnetic decays containing charmed quark-antiquark pair, physics simulation and event reconstruction at NICA.
\end{abstract}

\section{Physics case}

The analysis of X, Y, Z states is of great importance. Their predictions are closely linked to existing and forthcoming data of running and planned experiments like Belle, BaBar, BES, $\mathrm{LHCb}$, NICA, PANDA, etc. Given the existing experience of model calculation, physics simulation and event reconstruction, the detailed analysis of structure of exotic states can be performed. This can be realized by using well known methods based on QCD principles as well as new proposed phenomenological approaches, which allow describing the structure of bound state of hadrons and exotics. The obtained results will shed light on the nature of $\mathrm{X}, \mathrm{Y}, \mathrm{Z}$ exotics, which are one of the most mysterious states in modern particle physics [14]. Chamonium-like exotics spectroscopy represents a good testing tool for the theories of strong interactions, including: QCD in both the perturbative and non-perturbative regimes, LQCD, potential models and phenomenological models. The experiments planned at NICA are well suited for testing these states. The facility will allow colliding heavy-ion beams with the luminosity $10^{27} \mathrm{~cm}^{-2} \mathrm{c}^{-1}$ and $\sqrt{\mathrm{s}}$ up to $11 \mathrm{GeV}$ and proton-proton beams with the luminosity up $10^{32} \mathrm{~cm}^{-2} \mathrm{c}^{-1}$ and $\sqrt{\mathrm{s}}$ up to $26 \mathrm{GeV}[5]$.

\section{Heavy flavour study}

Good tracking and particle identification performance of NICA SPD over significant fraction of the final state phase space can provide a good opportunity to extend its ambitious physics program to studies of heavy charmed objects via their decays to electrons, hadrons or photons. It can be illustrated by the PYTHIA8 [6] simulated data on heavy quarkonia production in p-p collisions at $\sqrt{s}_{\mathrm{s}} \sim 26 \mathrm{GeV}$. One can see from Fig. 1 that the detector acceptance for electron-positron pairs from $\mathrm{J} / \psi$ decays is quite high $(\sim 80 \%$ for $\eta \mid<1.5)$. The detector acceptance for photons from electromagnetic decays of heavy quarkonia can be seen in Fig. 2. It exceeds $80 \%$ for $\chi_{\mathrm{c} 1}$ and $\chi_{\mathrm{c} 2}$ decays. The momentum resolution for electrons and photons is presented in Figs. 3, 4. One can see that it will be possible to combine momentum measurement of electrons in tracking detectors with energy measurement in the EMC to obtain better accuracy for fast particles. Signal reconstruction performance of the detector for $\mathrm{J} / \psi \rightarrow \mathrm{e}^{-} \mathrm{e}^{+}$decays was evaluated using full 
simulation/reconstruction chain. As can be seen in Fig. 5, J/ $\psi$ invariant mass peak is clearly visible.
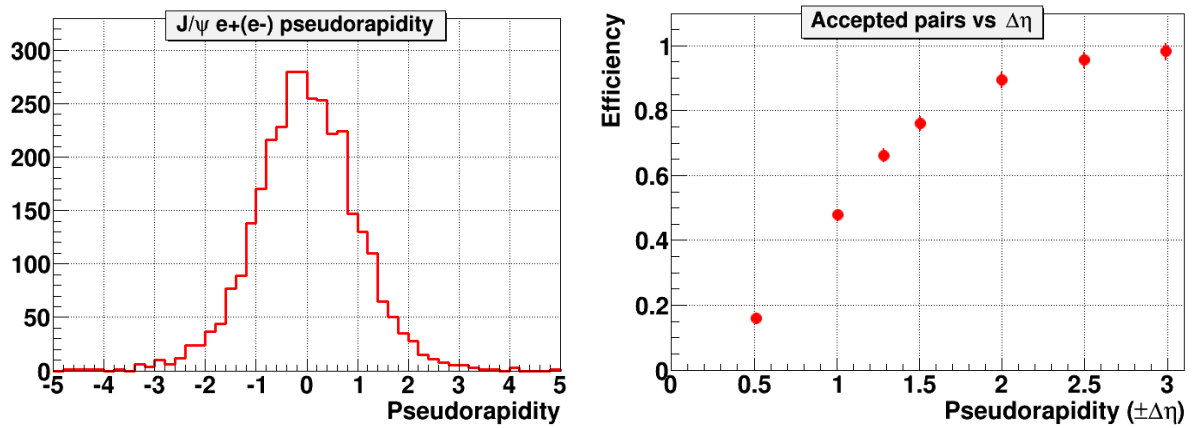

Figure 1. Left) Pseudorapidity distribution of electrons and positrons from $J / \psi$ decays. Right) percentage of $\mathrm{J} / \psi \rightarrow \mathrm{e}^{-} \mathrm{e}^{+}$decays with both the electron and positron within the pseudorapidity window of $\pm \Delta \eta$
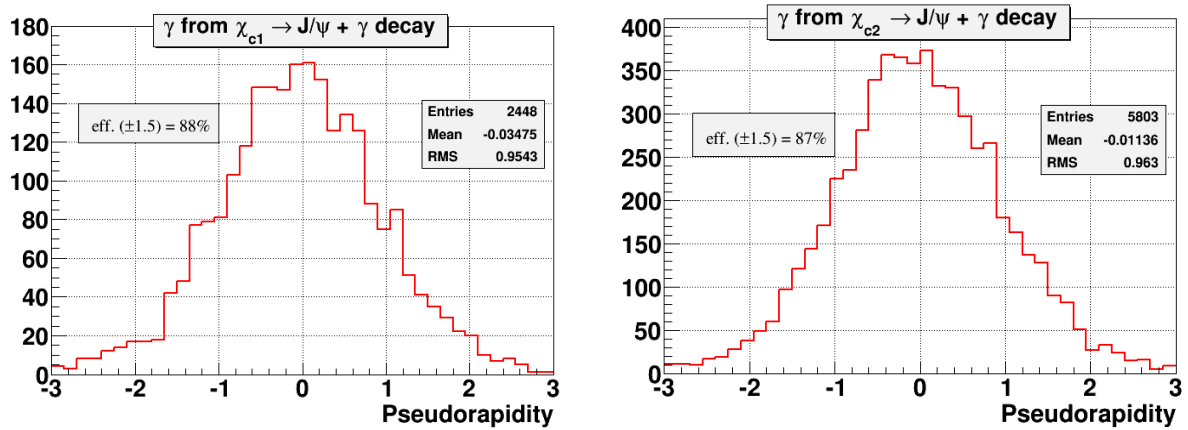

Figure 2. Pseudorapidity distributions of the photons from charmonia decays: left) $\chi_{\mathrm{c} 1}$, right) $\chi_{\mathrm{c} 2}$
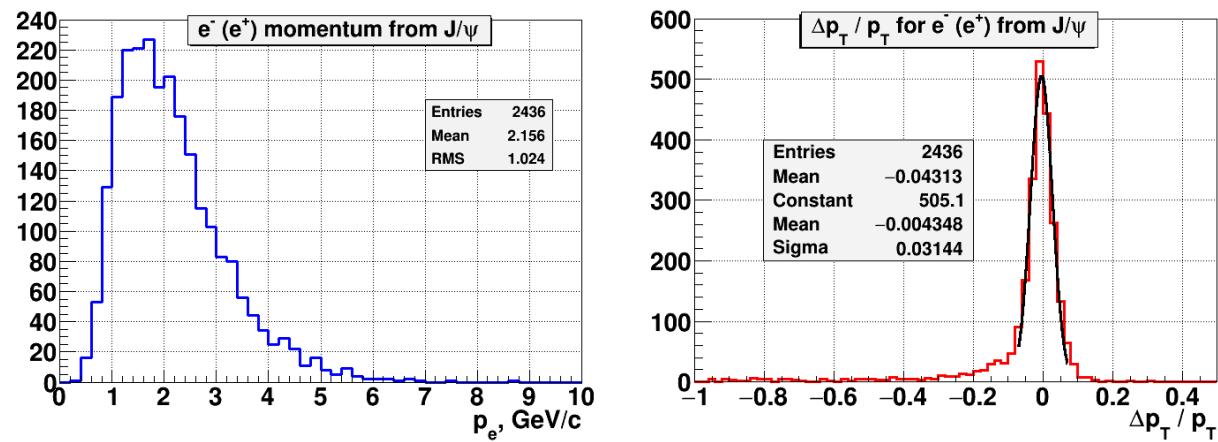

Figure. 3. Left) Momentum spectrum of electrons and positrons with $|\eta|<1.5$ from $J / \psi$ decays. Right) $\Delta \mathrm{p}_{\mathrm{T}} / \mathrm{p}_{\mathrm{T}}$ distribution for decay products, where $\Delta \mathrm{p}_{\mathrm{T}}$ is the difference between reconstructed and true transverse momentum 

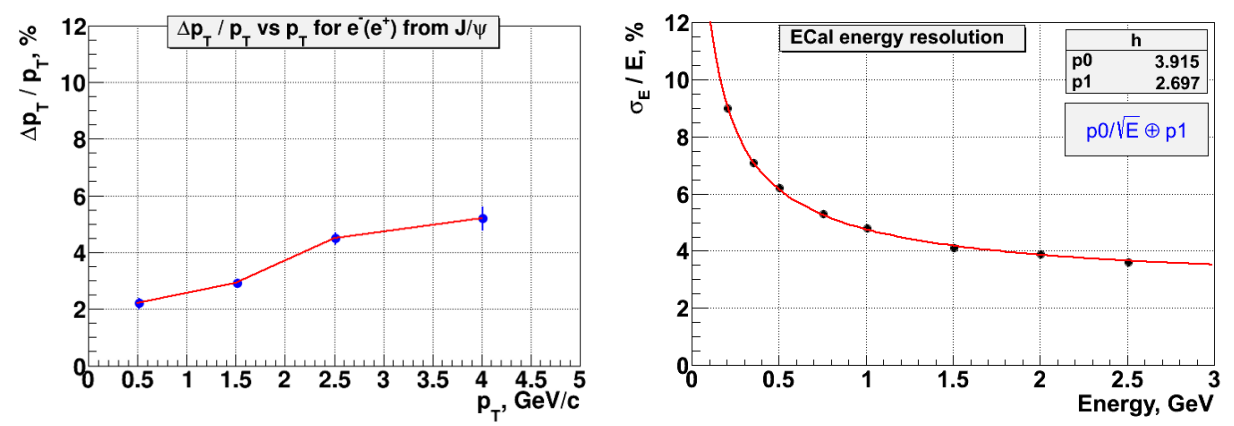

Figure 4. Left) $\Delta \mathrm{p}_{\mathrm{T}} / \mathrm{p}_{\mathrm{T}}$ versus $\mathrm{p}_{\mathrm{T}}$ for electrons and positrons with $|\eta|<1.5$ from $\mathrm{J} / \psi$ decays. Right) energy resolution $\left(\sigma_{\mathrm{E}} / \mathrm{E}\right)$ of the EMC for photons and electrons as a function of their energy
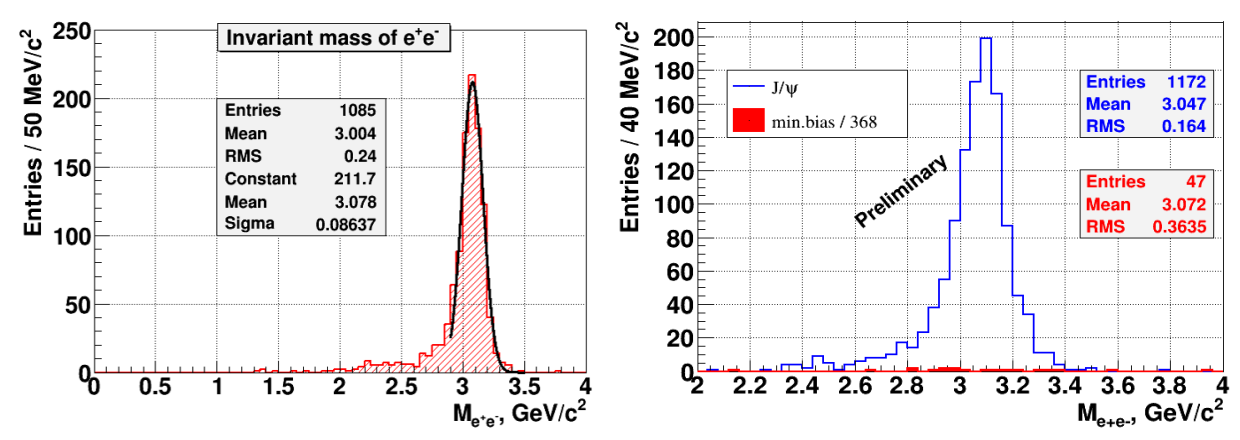

Figure 5. Left - reconstructed invariant mass of electron-positron pairs from $\mathrm{J} / \psi$ decays (signal); right - the same and minimum bias events (background), remaining after applying selection cuts (red). Note that only $1 / 368$ part of all minimum bias statistics was processed

\section{Reconstruction of $\mathrm{X}(3872)$}

The exotic state $\mathrm{X}(3872)$ was simulated in PYTHIA8 under the assumption that it is a charmonium state and the branching ratio to $\mathrm{J} / \psi+\rho^{0}$ was taken to be $5 \%$ [7]. As a result, the $\mathrm{e}^{+} \mathrm{e}^{-} \pi^{+} \pi^{-}$final state branching ratio $\sim 3 \times 10^{-3}$ gives the cross section for this channel of $12.2 \mathrm{pb}$ or $\sim 10$ days of running time at the luminosity of $10^{32} \mathrm{~cm}^{-2} \mathrm{c}^{-1}$ to produce $\sim 1000$ events. To better distinguish the signal peak from the background, it is better to use the invariant mass combination $\mathrm{Me}^{+} \mathrm{e}^{-} \pi^{+} \pi^{-}-\mathrm{Me}^{+} \mathrm{e}^{-}$due to its smaller width $(\sim 10 \mathrm{MeV}$ in our case as can be seen in Fig. 6). Fig. 6 also shows the background from events with charmonia production. The plots correspond to statistics collected during 10 months at luminosity of $10^{32} \mathrm{~cm}^{-2} \mathrm{c}^{-1}$. After fitting the background to the polynomial function using side bands of invariant mass distribution and subtracting it from the original distribution it is possible to observe a clear peak from the X(3872) decay (Fig. 7).

As an extension of this topic one can consider looking at other decay modes $\mathrm{X}(3872)$. Since the branching ratio of $\mathrm{X}(3872)$ to pairs of $\mathrm{D}$-mesons is much higher $\left(\mathrm{D}^{+} \mathrm{D}^{-}\right.$is $\sim 40 \%$ and $\mathrm{D}^{0} \mathrm{D}^{* 0} \backslash$ bar is $\sim 55 \%$ ), one should try to evaluate the possibility to reconstruct this state from hadronic decays of D-meson pairs. For such a study, the ability to tag the D-meson decays using the silicon microvertex detector should be very important. Thus, this physics topic becomes synergetic to the heavy ion charm program of NICA. 

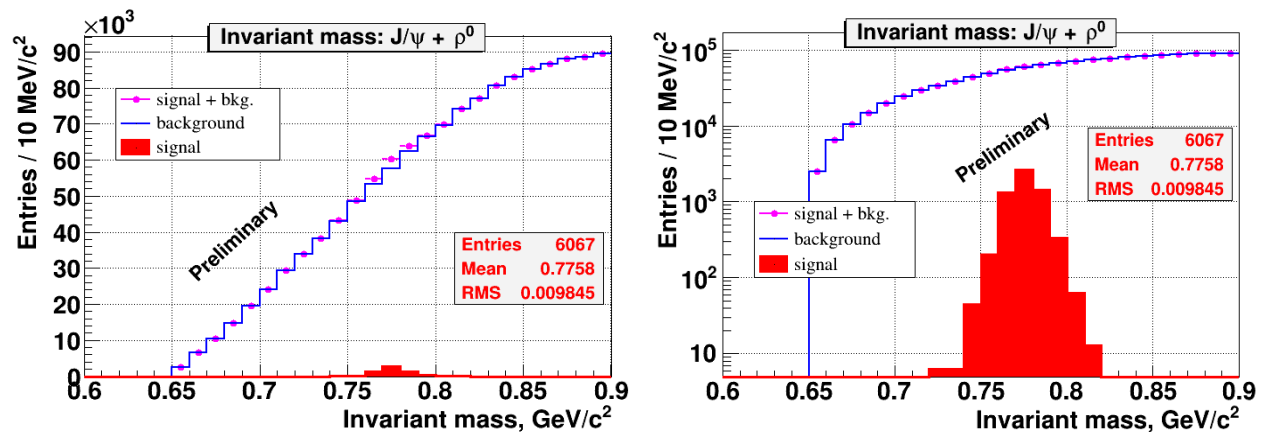

Figure 6. Invariant mass combination $\mathrm{Me}^{+} \mathrm{e}^{-} \pi^{+} \pi^{--} \mathrm{Me}^{+} \mathrm{e}$
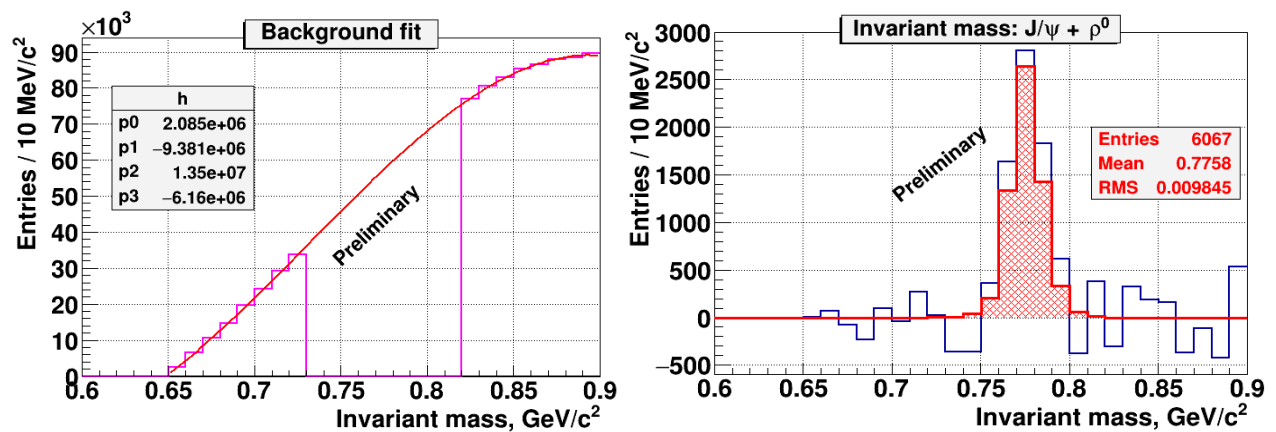

Figure 7. Left - Background estimation using the polynomial fit of the side bands of Fig. 6 (left). Right - background - subtracted invariant mass combination from Fig. 6 (left) (blue line) and true $\mathrm{X}(3872)$ histogram (red line)

\section{References}

1. N. Brambilla et al., European Physical Journal C 71, 1534, 1 (2011)

2. C. Patrignani et al. (Particle Data Group), Chin. Phys. C. 40, 100001 (2016) and 2017 update

3. M.Yu. Barabanov, A.S. Vodopyanov, S.L. Olsen, Physics of Atomic Nuclei 77(1), 126 (2014)

4. M.Yu. Barabanov, A.S. Vodopyanov, S.L. Olsen, A.I. Zinchenko, Physics of Atomic Nuclei 79(1), 126 (2016)

5. http://nica.jinr.ru/files/NICA CDR.pdf

6. http://home.thep.lu.se/ torbjorn/Pythia.html/

7. R. Aaij et al., LHCb Collaboration, Eur. Phys. J. C 73, 2462 (2013) 